\title{
Extended Range Guidance System for the Teleoperation of Microtunnelling Machines
}

\author{
A. Jardón, S. Martínez, J. G. Victores, C. Balaguer \\ Roboticslab-University Carlos III of Madrid, Spain \\ E-mail: \{ajardon, scasa, jcgvicto, balaguer\}@ing.uc3m.es
}

\begin{abstract}
-
Microtunneling is a trenchless construction method that is highly adequate to install pipelines beneath roads, rail roads, dams, harbours and environmentally sensitive areas. Microtunneling could be understood as a remotely-controlled, guidance operation of a MicroTunneling Boring Machine (MTBM), where references are provided by a human operator from the surface. A review of current technologies and main working principles of traditional guidance systems used to determine the position and orientation of the drill head of the MTBM during tunneling will be presented. Practical limitations and drawbacks will be discussed. A special attention will be given to those systems based on a laser-generated reference. In this paper a new video target system developed to improve the current performance of guidance systems will be presented. As it will be detailed, improving target processing of laser' incidence allow us to extend the minimum range to achieve a distance over $400 \mathrm{~m}$, without having to displace the total reference stations which are guiding the path during tunnel execution. The new target sensing principle, the implementation approach, the image processing and pose estimation algorithms will be discussed. Additionally, some preliminary results of the prototype in its current testing phase in real scenarios, gathered in parallel with commercial units, and its comparison will be presented.
\end{abstract}

Keywords -

Microtunneling machine, guidance systems, graphical interface, teleoperation.

\section{Introduction}

In order to successfully perform the teleoperated micro-tunneling machine drilling without excessive outof-tolerance alignment errors and without incurring cost overruns, the performance of the guidance system is crucial $[1,2]$. TBM's shield is articulated and can be guided by the orientation cylinders placed between the first and second shield. Traditional guiding systems are based on a reference laser that marks a straight line to determine the position and orientation of the drilling head during tunnel construction. Also, this target requires complex oriented prisms systems for the estimation of position and orientation of the machine. All of this leads to delicate equipment, with complex handling and installation and which leads to higher construction costs.

The guiding system which will be described allows knowing the orientation and positions of the tunneling machine based on the capture of the incidence of a green laser on a custom-made video target. With this, the working range of the guiding system increases, avoiding having to relocate it, minimizing the impact in time and therefore, costs when drilling with it.

\subsection{A brief review of existing guidance systems}

The importance for the final results of the excavation pushes the ongoing improvement of target systems. When exploring Patent databases, several guiding methods for tunneling machines can be found. In [3] a method is designed based on a target with two detectors for the beam of light. A first detector is a semi-translucent mirror that deviates part of the beam to one side of the machine, while allowing another part of the beam to pass. Then a second detector is located at a known distance, following the same method. On the sides are two light detectors with photosensitive plates, to obtain the incidence position of both beams. From those deviated light points inside the target, the position of the machine is calculated against a laser reference in a general purpose computer. This way, the machine is guided by a laser beam that marks the orientation and slope of the drive.

Commercial guiding systems based on similar working principles are Gyro Navigation Systems Laser Referencing [4] or Laser based system [5]. An example of commercial gyro based is one that uses a fibre optic/mechanical gyro mounted within the TBM [6]. The Gyro System is best for small diameter drives (ID $800-1,200 \mathrm{~mm}$ ) or for drives with muck skips and bounded views inside the TBM. A Gyro-based Guidance System uses a self-levelling, north-seeking 
gyro compass for the determination of the horizontal position and azimuth of the TBM. The calculations of these systems are based on the principle of the dead reckoning, which means that it is assumed that the TBM will move exactly along the direction which is defined by its axis. The difference between the direction along which the TBM is moving and the axis of the tunnel is called the TBM drift. This drift will influence the calculations of the system. Check measurements for the calibration of the system must therefore be carried out every 20-40 metres in order to determine the TBM drift and reduce its influence to a minimum by pre-setting this value for further calculations.

For a curved drive the choice of commercially available guidance system is either a Laser based system or a Gyro Navigation System. Laser based guidance systems have been used for many decades. Traditionally, a laser station is firmly fixed inside the tunnel, projecting a laser point onto a laser target board mounted on the TBM, as shown in Figure 1. A target that is sensible to a red laser $(635 \mathrm{~nm})$ is mounted on a TBM as an aiming unit, with high precision motorized prisms, for electronic measures of distance mounted on the same vertical axis of the target's centre. Based on the offsets of the laser spot on the target board, the TBM operator infers the current line and grades the tunnel alignment deviations. A laser emitter is located on the fixed platforms on the back side of the tunnel. The laser beam has to be obstacle free to be able to impact in the sensitive target. Sometimes, this simple laser emitter is replaced by a Theodolite total laser station and prism for further referencing. The Theodolite is equipped with a sensory system that allows the automatic aim of the prisms. Last, a prism acting as a previous aim of reference located on the previous platform where the Total Laser Station is located. It works as a visual orientation of the total station.

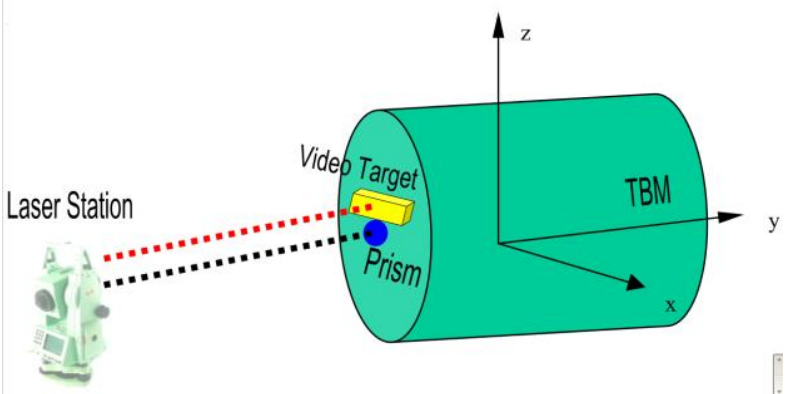

Figure 1: Laser target referencing.

Following a straight line, the distance between the platforms is normally between 30 and 100 meters, which is the minimum advised distance for installing the platform from the target, because with a lower distance, the machine thrust forces generate movements in the concrete segments which would lead to lack of precision in the guiding task. Likewise, the maximum advised distance so the laser beam refraction errors do not affect the precision is 100 meters.

Examples of these commercial systems are the ELS Electronic Laser Systems Basic System or Video Target based systems. An example of the first could be found from ZED Tunnel Guidance Ltd [7]. Another ELS based commercial systems are SLS Microtunneling LT guidance systems supplied by VMT GmbH [8]. The TACS GmbH company also offers video target based systems [9]. These are guiding systems for tunneling machines based on the incidence of a laser on a target sighting, designed for tunnel alignment in which only big curvature radius can be produced. Those systems are based on sensitive panels, fixed to the TBM body, and on the usage of an inclinometer. The sensing principle of TACS' video targets has inspired our design. It consists of two target boards and two cameras inside a robust housing (IP68) in several measures [9]. The video targets determine the coordinates of the laser hit point by means of high precision video cameras and proprietary recognition software.

\subsection{Drawbacks and limitations of current guidance systems}

Traditional guidance systems are based on several modules working over a laser-generated reference (red light, 620-640nm). However, the first factor that limits the accuracy and reliability of traditional laser guidance systems are the working environment. Tunnels' condition of dust is a limiting factor for the maximum range of the guidance system. Dispersion and refraction of the laser beam over a long distance make difficult for a laser beam to impact the photo-electronic sensible panel of the front side on the target. The second limitation is related with the geometry of the projected tunnel: sudden changes in tunnel axis direction have been avoided to limit the point charges on the exterior edges of the pipe, and to prevent damage in joints. Abrupt changes in direction would also bring as a consequence great frictional resistance against the previous pressing of the complete pipe length, which could only be solved by especially elevated pressure loads. The more different the trajectory compared to the plan, the more pressure on each section of the tunnel and ground settlement in the area. In the case of concrete segment construction systems, coupled behind the microtunneling machine, the drilling heads transmit vibrations and movement on the terrain under the concrete segments and between these same concrete segments, which affect the inertial instruments and the positioning of the reference base stations when moving forward.

The third limiting factor is related to the fixation platforms of aiming lasers. Although concrete segments 
are manufactured according to the original plan, they are not exempt from small relative movements, which can be sources of error if not considered in the calibration phases. As soon as the tunneling machine and the length of pipe are so far ahead that the laser beam can no longer reach the target from the access chamber, the laser should be mounted on a console, in a proper position behind the target, fixed in the length of pipe. This assumes that with the traditional systems, every 30 to 100 meters the laser or prisms should be properly relocated to achieve the guiding task, which creates a sizeable delay and a lowering in the total machine's productivity, due to the big performance that tunneling machines offer (drilling up 40 meters per day).

The fourth limiting factor lies in potential manual errors in initializing or calibrating the laser beam's alignment. Although human factors could be avoided by providing more automation to the guidance system, most laser systems suffer difficulties to receive laser's projection because of excessive TBM deviations. Current ELS targets have been in reduced dimensions because the cost of photosensitive cells is very important. This leads to having a very small laser window (typically 100x100mm). Therefore, in case of machine deviations, where the laser window is missed, the position of the laser should be changed to locate it again on the laser window. For the same costs, a Video based target with optical cameras with bigger dimensions can be constructed.

\section{Design and development of a novel vision based target}

To improve the limitations of the current guiding systems, what is needed is a guiding system that combines the classic concepts of tunnel survey in an optimal manner, against the operation costs, achieving a guiding system that: a) Shows immediate information of the tunneling machines against the designed axis, offering an excellent precision, normally above the driving features of a tunneling machine. The tunnels are normally built with a few centimetres of error in the path, but this is caused by the impossibility of driving the tunneling machine exactly through the indicated axis given by the guiding system. b) Does not interfere on the progress or forward movement capacity of the machine, for having to reposition and recalibrating continuously the stations and reference prisms, or relocating the aiming target in relatively short time intervals, to allow monitoring the tunnel's construction.

\subsection{Functional architecture}

In order to achieve the above goals, improvements in subsystems, the laser target, and the user interface have been implemented. A low cost reliable guidance system has been designed for any type of TBM (e.g. pipejacking or concrete segments) based on a new laser type and target combination. The target has been totally designed from scratch to allow sensing the laser beam inside, e.g. has gotten two surfaces where the beam incidence is capture by a vision system. As described in figure 2, the first surface is located in the front window, which is semi-transparent. The other surface is the interior back panel of the target. The inner walls of the target space are black painted and the cameras and on board electronics are located on the bottom. The front panel is made of a methacrylate panel with an embedded $1 \mathrm{~mm}$ clearance net (grill).

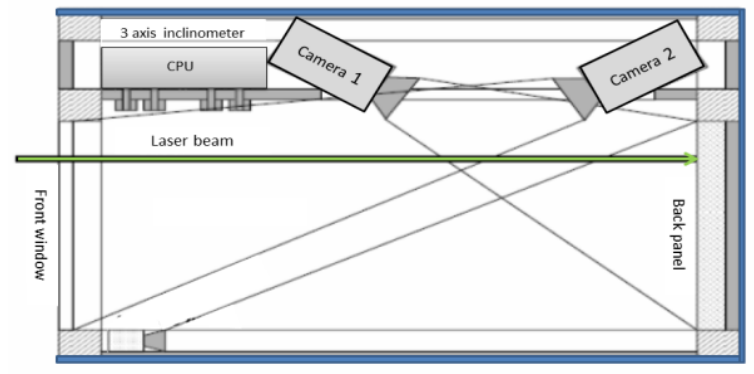

Figure 2: Functional layout of the novel vision target device.

\subsection{Implementation of beam incidence capture system: Vision target}

The new video target developed is sensitive to green laser $(532 \mathrm{~nm})$ instead of red laser as in commercial targets. It performs high resolution image processing and simple but reliable pose location algorithms. To complete the design of a vision based guidance system, this video based target has to be integrated in a modular guidance system that could be mounted in several types of machines with few adaptations.

The general architecture has three parts. The first is the smart target, which is the remote equipment (right part), where we have the target formed by the cameras, embedded computer, and the encoder (drawn in the left part of the image) for odometry. The encoder block is optional. In this module the image capture and processing modules will run obtaining the information from the inclinometer. The second part is formed by the local equipment in the command cabin, which executes the graphic interface, implemented in a computer with a touch screen. To connect them a wired or wireless data link allows the exchange of data to the guiding interface and the smart target. This block runs the representation system and the information representation relevant to the guiding process, in a touch screen. Here the data provided by the calculation module are represented and an interaction with it can be made. Also the communications module will be executed where the 
protocol and the transmission method for the processed information will be established for several tunneling machine environments. The third part, which is essential, is a green colour laser emitter $(532 \mathrm{~nm})$, where the axis has been properly positioned by using references to the base station, by traditional topographic methods. The complete system can be seen on figure 3 . The visual system has been developed under the premise to use a green laser emitter, because it has a longer range and less dispersion problems than a red laser $(635 \mathrm{~nm})$.

The on-board electronics of this remote system is based on low-cost robotic hardware and software technologies. The core of the system a low-cost embedded "ROBOARD", a 32-bit credit-card sized x86 CPU, widely used to develop low cost robotic applications. This CPU [10] manages two USB cameras and the inclinometer unit. It is fully compatible with $\mathrm{x} 86$ libraries and its cost is about $200 €$. Image acquisition is performed by two low-cost (about $40 €$ ) and simple USB Logitech Quickcam Pro 9000 (V-U009), working at 2MP resolution. OpenCV computer vision libraries [11] and YARP middleware [12] manage the image vision and wired/wireless data link to the tunneling machine control station (normally on surface and not underground, except in open shielded TBM), where a local PC running a GUI application allows to get

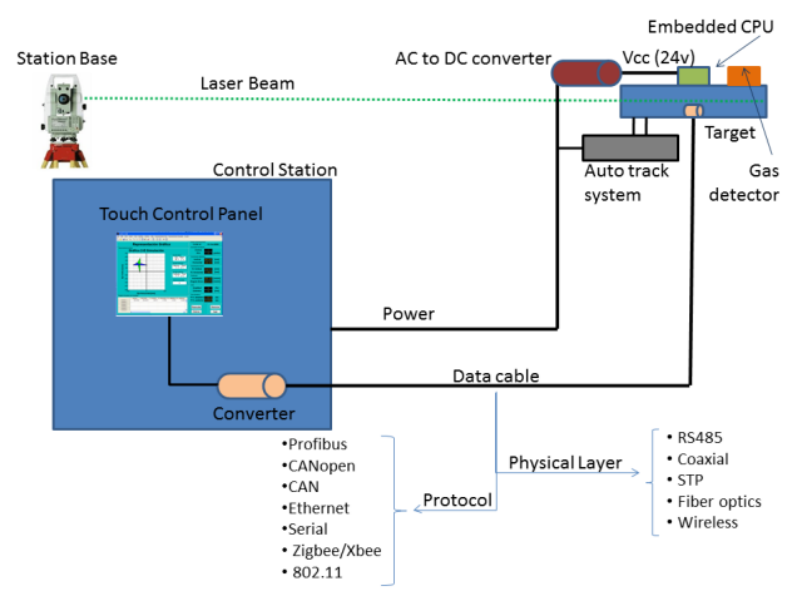

machine location data on execution time.

Figure 3: Hardware architecture of the new guidance system.

The new video target, which has external dimensions of $700 \times 225 \times 160 \mathrm{~mm}$ and beam reception windows of $170 \times 170 \mathrm{~mm}$, is installed with a clamping support to the tunneling machine, mounted on its higher panel. The guiding computer fulfils the protection standards IP65/NEMA4, indicating it is protected against dust and low pressure water, which is expected to suffer the most severe environment conditions and should be properly protected for its usage. The system's power is $220 / 230 \mathrm{~V}$ in a guiding cabin for the GUI computer and $9 \mathrm{~V}$ DC $(10 \mathrm{~W})$ for the target module. They are joined by a UTP C5 network communication cable with military RJ-45 connections. About $15 \mathrm{~m}$ of cable were used as a necessary length. In order to make the image processing from the cameras more robust, a notch High Performance Laser-Line Filter, from Edmund Optics of the $12.5 \mathrm{~mm}$ diameter model, was installed in the back camera pointing towards the translucent window, getting rid of all the frequencies except the laser one with a wavelength of $532 \mathrm{~nm}$ and bandwidth of $2 \mathrm{~nm}$. This eliminates the problem of obtaining an image with excessive exterior tunneling machine light.

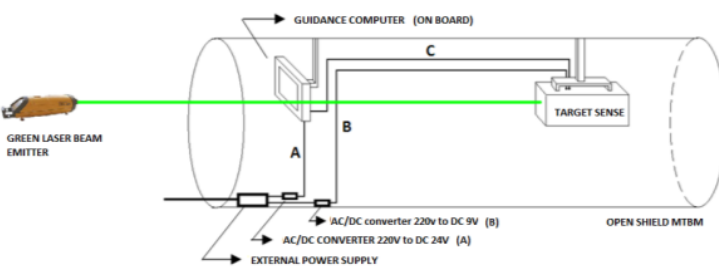

Figure 4: System Setup for UC3M's guidance system.

The use of open source libraries and low-cost, but rugged hardware approach, make an increased reliability with respect of expensive commercial systems possible, allow a more simple operation and setup phase, and incur in lower operational costs. The most expensive piece of equipment is the tactile panel due to the high IP code requirement required for an underground environment. However, this unit could be removed thanks to the data link implementation, which allows operating the system from virtually anywhere.

\subsection{Vision algorithms for beam incidence capture}

Once the image is captured, the next step is to process it to determine the central point or centroid, which represents the laser beam hitting the panel. The problem in these conditions is to obtain coordinates from the centre of the object that would be detected as the laser beam. It should be simple, because it basically shows a black image with a single point caused by the laser. The vision target's interior walls have been painted in matte black. This makes easy the image recognition. However, the translucent panel causes more problems. This panel is subject to external environment conditions, either because of the luminosity provoked by the luminaries in the interior of the machine and that would be impossible to hide to the target, or the filth and water in the environment. For these problems, the window has a net in the translucent panel and a notch 
filter to recognize only the wavelengths close to the ones from the laser of $532 \mathrm{~nm}$ for green light.

The fundamental problem to precisely recognize the incidence point is to discriminate between the different points found on the image, which can be either the desired laser point, as reflections produced by the laser or the external illumination, which is why the image has to be treated to separate this point from the others, and even if the laser beam is lost, provoked by situations like a material or a worker is on the laser's trajectory or it falls out of the range, the program detects it and generates the corresponding message.

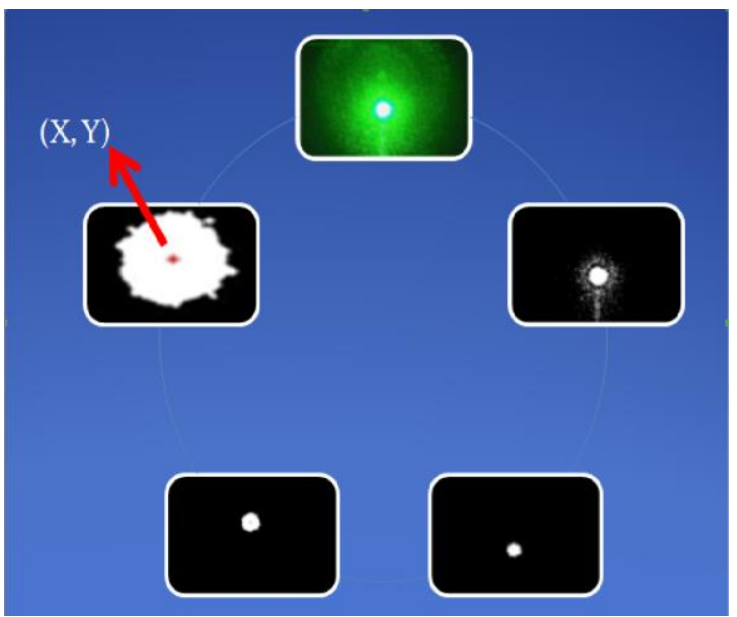

Figure 5: Simple laser incidence image processing.

The image processing, once loaded, binarizes the image based on a threshold, searches on the image all the possible objects with a circular shape that are within a characteristic diameter (because the laser a beam of $12 \mathrm{~mm}$ nominal diameter) and calculates the centroid of the given object. In case no object with such characteristics is detected, this could be because the threshold value is too high, thus eliminating even the point generated by the laser, reducing this threshold a fixed value and reproducing the previous algorithm over and over until it looks similar to the one needed. In case it detects several objects that do fulfil these characteristics, having reached the binarization threshold (where it doesn't detect the laser incidence), the biggest areas are chosen as acceptable points, communicating to the guiding employee there could be some miscalculations caused by excessive light on the target. From the point obtained as the laser beam, the program communicates $\mathrm{X}$ and $\mathrm{Y}$ coordinates as well as longitudinal deviations produced in this image plane, diameter of the detected object and number of possible laser beam points found in the last program's iteration, in case a possible error in the beam detection needs to be notified [13].

As shown on the figure 5, for the proper measurement of the incidence of the laser in the frontal window, a camera with a notch filter attached to it has been used so it allows only the green laser frequency $(532 \mathrm{~nm})$ to pass. Despite the fact that it lowers the amount of perceived light, it improves obtaining the centroid of the image, because reflections and light sources that could incur in false estimations of the incidence position of the beam in the frontal panel are removed. To convert the information of the images of the laser incidence on the target's back window (frontal incidence image) and the back panel (back incidence image) from the orientation of the machine, the installation position of the target against this and the inclination of the machine against its forward axis (roll) should be known. A low cost but precise inclinometer can be used for this purpose. Knowing the fixed location of the target with respect the tunneling machine, the next section explains how to calculate the machine's orientation with respect to the laser line, and the machine location with respect to the laser base location.

\subsection{Position and orientation estimation}

Here a brief description of the referenced setup while drilling and associate references will be presented.

Initially the system is calibrated by using the laser beam reference against the coordinate system of the topographic system. Likewise, the target is located at the beginning of the tunnel with the Theodolite topographic laser station.

When initializing the localization algorithm, the following reference systems (coordinates systems or SDC) between the different parts of the guiding task are considered:

a) $\mathrm{SDC}_{\mathrm{w}}$ : World coordinates system, fixed to the entrance of the attack well as a geo-localized global reference system. 

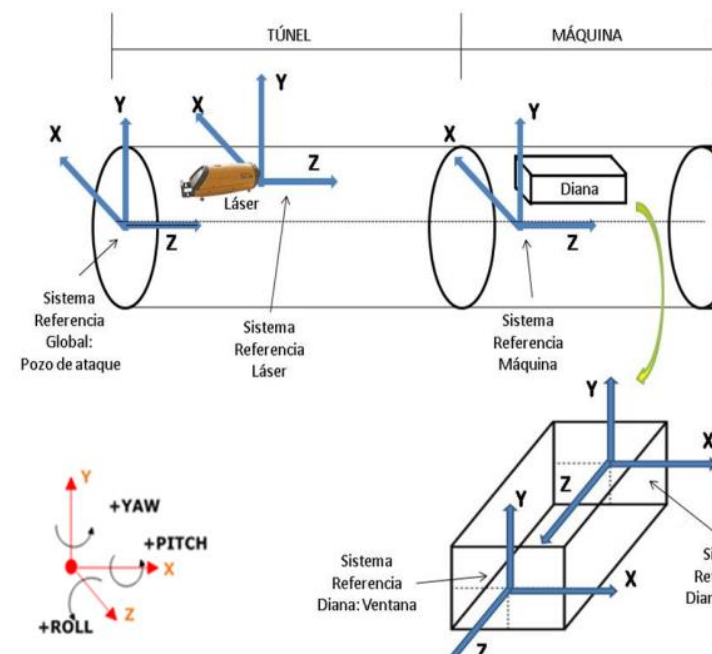

Figure 6: Coordinate frames for location algorithm.

b) $\mathrm{SDC}_{\text {Laser }}$ : laser position coordinates system, which will be fixed as a reference for the aiming system that will calculate the position of this laser beam, but that will be relocated when its range surpasses the longitudinal reaching limits of the beam (normally under $500 \mathrm{~m}$ for a green color laser) or when the tunnel's radius of curvature geometrically prevents it from reaching the aiming system being used.

c) $\mathrm{SDC}_{\mathrm{EPB}}$ : reference system to express the tunneling machine's coordinates against the $\mathrm{SCD}_{\mathrm{w}}$ coordinates system, as the main output of the system.

d) $\mathrm{SDC}_{\text {target }}$ : in current commercial systems, located at some point of the traditional target, typically in the centre of the laser's detection panels. Commercial systems only use an incidence plane and the SDC is fixed. Therefore, it is fixed from installation and should be referenced when the machines starts, by either the topographer or the machine's manufacturer.

Once the coordinates in the image of the laser beam have been obtained, they have to be referenced to the same coordinates in millimetres above the central point of the image as the origin of the coordinates. This is a typical pose estimation problem well modelled by the proper SDC. It has been identified that it is then possible to solve the pose transformation from a set of equations which relate the SDC orientations of the rear and front panel, with their 2D image coordinates, taking into account that both are parallel planes. To do this, a $\mathrm{C}++$ class has been developed that creates simultaneous equations relating the corners of the images as points of known coordinates, generating a fixed calculation matrix that, multiplied by the coordinates obtained by the vision system, obtains the coordinate system that transformed finally to the deviations from the origin of the tunneling machine as a solution.

The details of the equations of calculation and composition of rotations and displacements are detailed in [13]. They have been programmed using MATLAB scripts and afterwards converted to $\mathrm{C}++$, so they can be integrated in the system software architecture. The modules of calibration and image capture, the inclinometer readings and other sensors (i.e. gases), and alarms (i.e. by incidence lost) are executed on the target. All the modules communicate using YARP that also runs on the pilot's touch panel. Here the GUI, the calculation module, Data Logger module and the windows for warnings and errors are also executed. The implemented architecture has the guiding system in the machine itself, performing the connection directly by Ethernet protocol. A standard twisted network cable has been employed, properly protected. In case the guiding screen would be located outside, the same structure would be kept but a physical media to interconnect the different systems at long distances would be needed.

\subsection{Pilot interface}

In order to successfully carry out a teleoperated microtunneling machine drilling layout plan, not only is important how to achieve the referencing but also equally important is how this information is presented to the pilot.

The size of the tunneling machine must be known, as well as the position of the target inside the given machine. Figure 7 shows the necessary setup data that enables a correct calculation of the software guiding trajectory. Using the size in $(\mathrm{mm})$ :

A: Total tunneling machine length (11000).

B: Diameter of tunneling machine (2000).

C: Distance from back of tunneling machine up to target's translucent window (8250).

D: Distance from target's translucent panel to front of the tunneling machine (2750).

E: Height from the central symmetry axis of the tunneling machine to target's longitudinal symmetry axis (21).

F: Horizontal distance from the central axis of the machine to target's centre (676).

The table of guiding records is important so the user that controls the tunneling process monitoring can do a full parametric tracing of each forward movement produced in the tunneling machine. This data is shown in the table in the lower part of figure 10. 


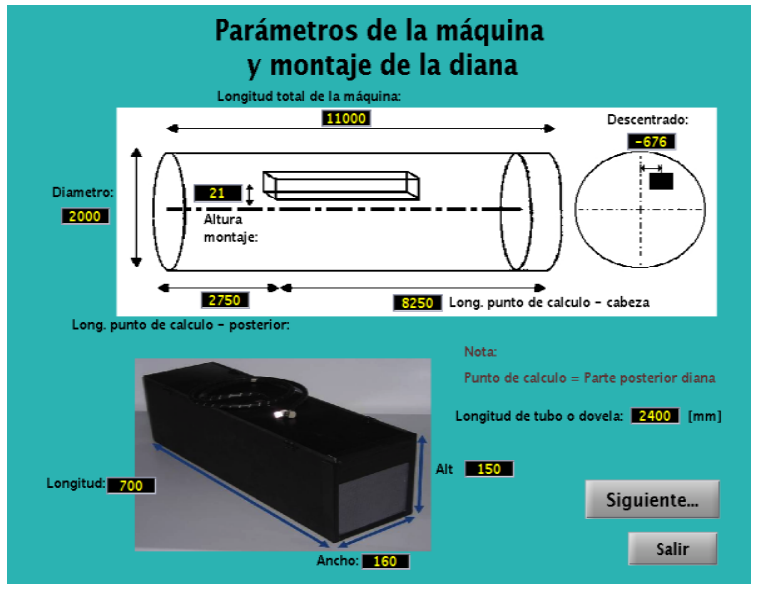

Figure 7: Pilot interface setup screen.

The main parameters to show in a guiding system are the horizontal and vertical deviations, and the called drift and tendencies. The deviations are the distances between the axis of the project and the axis of the tunneling machine, measured in the perpendicular plane to the first. The tunneling machine is usually presented as an arrow and the deviations are presented in two points of this axis, the front and the back. These deviations are given every time a position is taken, because when giving coordinates of these points, they are compared against the path stored in memory. One of the most important parameters for the pilot is the tendencies. The tendencies indicate the movement that the machine would have after a forward movement, giving an idea of the scope of the correction being applied. The pilot guides the machine exert certain pressures in the guiding cylinders and in the using ones, and these pressure values are taken by the program to calculate tendencies.

Another improvement of the systems relies in the user interface, in which that information is displayed and updated dynamically with the movement of machine. In the following section, a new way to display the position of the machine using a 3D representation is presented. This way, the pilot responsible for the operation can understand better where the machine stands and what the following actions to perform on the control of the machine to get take on the planned route are.

\section{Experimental results}

The preliminary results of the prototype in its current testing phase in real scenarios were obtained at a workplace where the guidance system was mounted into an open-shield boring machine. From this moment, the TBM started boring in the field workplace. Due to existing limitations and the impossibility to alter the machine's production rates, the tests were performed in parallel with a commercial system. Our developed system was mounted in parallel with the EUROHINCA proprietary system based on a customised VMT's SLS Microtunneling LT system, according to the employed machine [8]. In the initial starting phase (Phase 1), the laser station, which comprises VMT's standard Leica TCA 1203plus motorized total station and which includes an integrated diode laser mounted parallel to the visual axis and a sensor system that allows automatic targeting of prisms, was mounted on a custom built measurement pillar between the jacking-pit thrust rams. The green laser diode emitter was mounted in parallel and also referenced. For reference measurement a further pillar with a survey prism was installed with a reference prism outside the shaft. The green laser used was a Topcon model TP-L4G, mounted and calibrated for its usage, powered with a battery. On the first stage, distance tests were executed at $50 \mathrm{~m}, 100 \mathrm{~m}$, and $200 \mathrm{~m}$ in the environment of the Collector Tunnel of Jalon River, a tunnel project with internal diameter of $2000 \mathrm{~m}$ and $479 \mathrm{~m}$ long [14]. After initial setup and verification, the first distance test was done at $50 \mathrm{~m}$.

Figure 8 shows the incidence of the beam in the front (up) and rear (down) window taken at $50 \mathrm{~m}$. The measurement taken at $200 \mathrm{~m}$ turned out to be complex, because it required relocating the commercial system in parallel and then calibrating it. Afterwards, without moving the green laser from its original position, the incidence of the green laser beam in the target was obtained, resulting in the images shown on Figure 8.
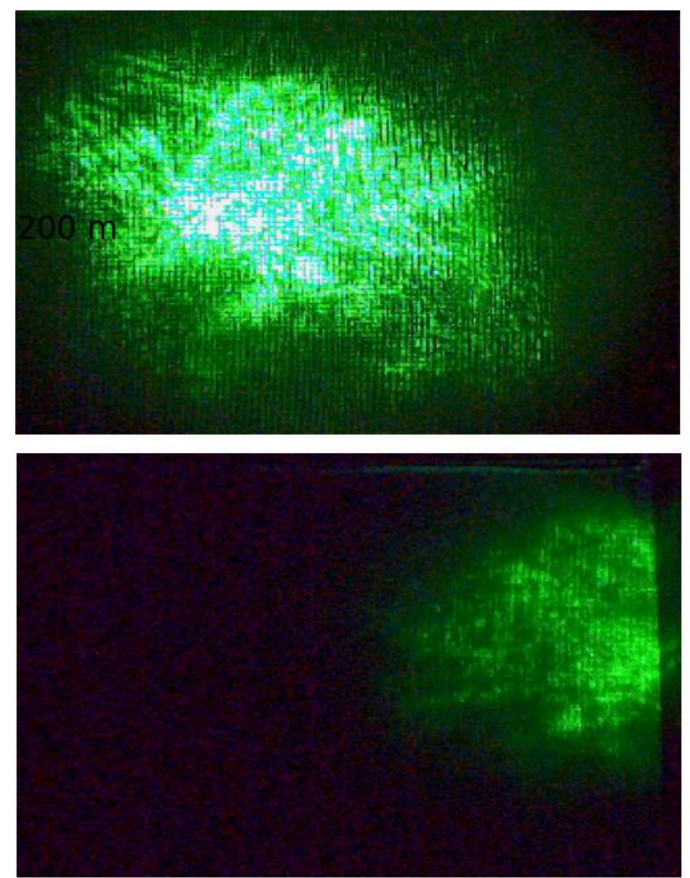

Figure 8: Front window view and rear window view at $50 \mathrm{~m}$. 
The Figure 9 shows the dispersion of the beam due to the distance and the presence of suspended particles because of the roadheader working. Even though during the test the resulting beam incidence is highly faded, thanks to the robustness of the vision algorithms, the data from the position of the target was obtained. Then, the data is shown by the guiding interface. In this case, and even though much dispersion exists, the algorithm could estimate the centroid of the zone of the greatest light intensity, meaning, where there is more incidence. Therefore, it indicates the beam's direction, which highlights this data as an improvement factor of current targets. This allows, for example, using conventional mirrors, to redirect the beam in case of incidence loss.

As shown on Figure 10, the main screen of the interface developed for its validation during work has been implemented on a touch PC with IP67 protection. The information is presented in such a way that the pilot comprehends the effects of his/her actions on the trajectory in a simple manner, because of the 3D visualization, and can understand the behaviour of the machine because of the actions and correct them in an easy and intuitive way. During excavation, the laser beam was maintained continuously on the ELS (electronic laser system) target during the TBM advance.
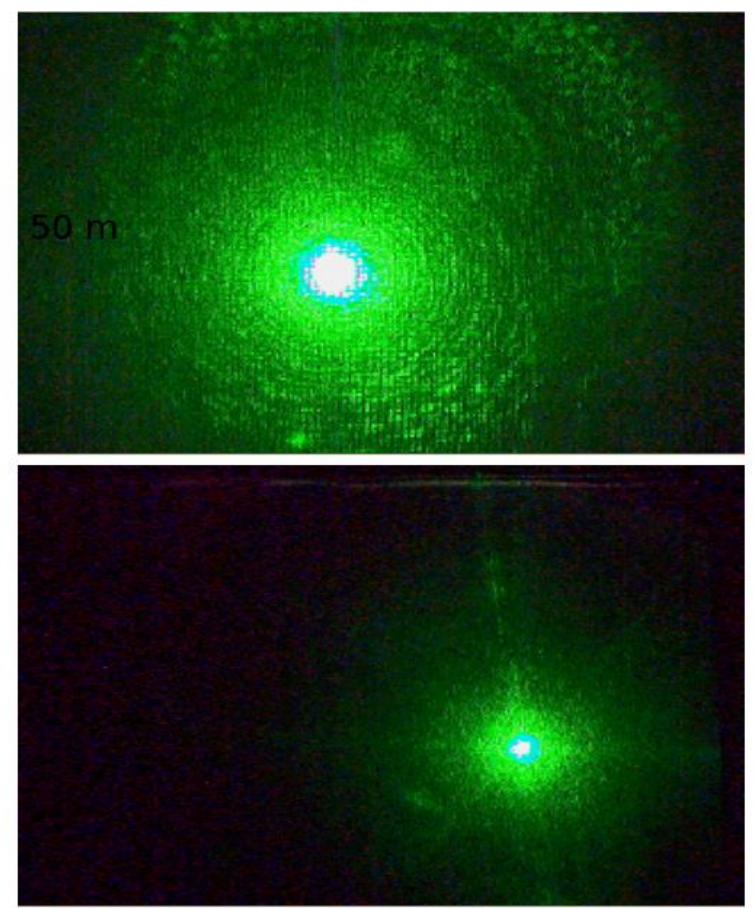

Figure 9: Front window view and rear window view $(200 \mathrm{~m})$

The calculated values of position are displayed on the system's computer screen and stored in the database. The three-dimensional coordinates of the axis point in the ELS target unit plane are compared and then saved as the projected course of the TBM measured by conventional procedures.

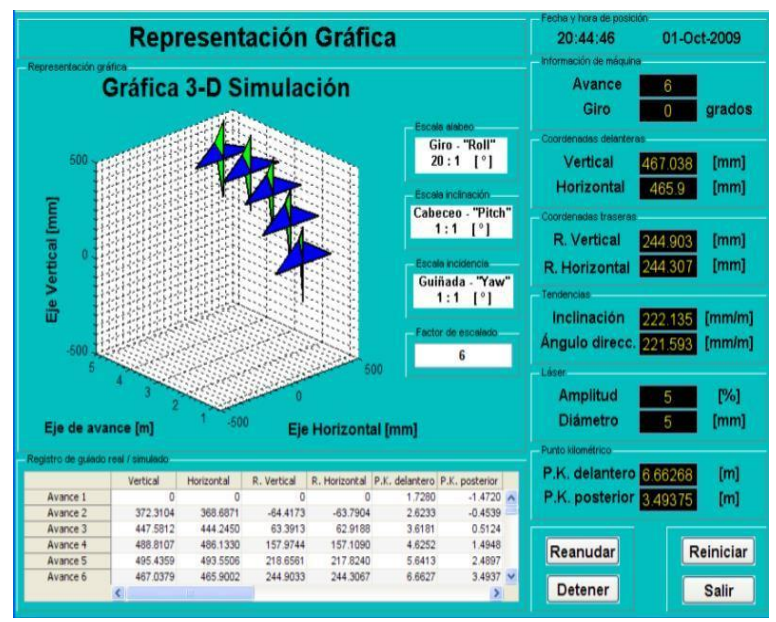

Figure 10: Guidance 3D path as showed in the GUI

For each instant, a calculation of estimated location of the TBM measured with our system was performed. In table 1, a comparison of deviations and estimated errors for both systems are shown. All the measurements obtain a $0.2 \mathrm{~mm}$ linear and a $0.1^{\circ}$ angular repeatability. As trueness in the measurements is close to precision values, a good accuracy is obtained. It is proven that the developed prototype reaches sharper precision values for the laser beam capture. This implies better capacity when obtaining the machine's position.

Table 1. Accuracy of field measurements

\begin{tabular}{|c|c|c|c|c|c|}
\hline & $\begin{array}{c}\text { Accuracy } \\
\text { HD mm }\end{array}$ & $\begin{array}{l}\text { Accuracy } \\
\text { VD mm }\end{array}$ & $\begin{array}{c}\text { Angular } \\
\text { accuracy } \\
\text { Alfa }^{\circ}\end{array}$ & $\begin{array}{c}\text { Angular } \\
\text { accuracy } \\
\text { Beta }^{\circ}\end{array}$ & $\begin{array}{l}\text { Angular } \\
\text { accuracy } \\
\text { Gamma }^{\text {o }}\end{array}$ \\
\hline $\begin{array}{l}\text { ELS1 } \\
\end{array}$ & 1 & 1 & 0,05 & 0,05 & 0,05 \\
\hline ELS2 & 1 & 1 & 0,05 & 0,05 & 0,05 \\
\hline $\mathrm{UC} 3 \mathrm{M}$ & 0,267 & 0,267 & 0,025 & 0,022 & 0,016 \\
\hline
\end{tabular}

The second table shows a comparison between the angles and deviations on current targets and the UC3M prototype.

Table 2 . Comparative of linear and angular maximum allowed drifts.

\begin{tabular}{ccccc}
\hline & $\begin{array}{c}\text { HD } \\
\mathrm{mm}\end{array}$ & $\begin{array}{c}\text { VD } \\
\mathrm{mm}\end{array}$ & Alfa $^{\mathbf{o}^{\circ}}$ & Beta $^{\circ}$ \\
\hline ELS1 & 115 & 60 & 10,95 & 20,35 \\
ELS2 & 146 & 88 & 15,84 & 15,84 \\
UC3M & 160 & 120 & 10 & 13,24 \\
\hline
\end{tabular}


Table 2 has the maximum horizontal and vertical deviations as well as the maximum angular pitch and inclination measurable with the designed target's prototype. Note that values are similar to actual models but should be considered the lower cost in the components.

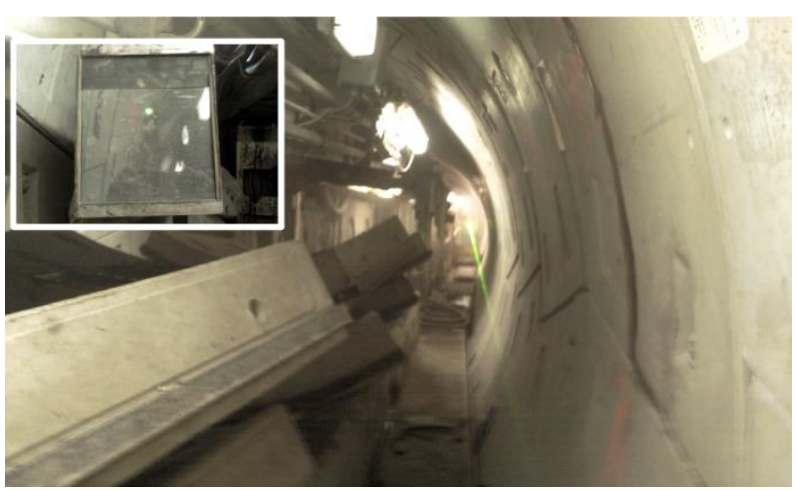

Figure 11: Guidance system working in workplace and laser incidence at GUI

It was estimated that a prototype can be implemented with bigger vision panels (about 250x250 $\mathrm{mm}$ ) and shorten the length of 700 to $400 \mathrm{~mm}$, to maintain the angular precision, having proved in situ that there is enough space allowing this application.

Note that the simple design of the target allows rebuilding it in custom sizes without great efforts, especially if the size of the vision panels has to be increased. This makes shortening the length of the target possible, to maintain the current angular resolution.

\section{Conclusions}

A modular a low-cost reliable guidance system for TBMs has been presented. The preliminary results of the prototype in its current testing phase in real scenarios (working in parallel with previous commercial units for comparison) were obtained in a real workplace where the guidance system was mounted on an openshield boring machine. Increased reliability has been achieved with respect to commercial systems, using low-cost embedded hardware with a Linux based O.S. and OpenCV libraries, allowing a wired or wireless data link to TBM control station (normally on surface and not underground). This low-cost approach is not only for development of the target itself, but also allows lower setup phase and operational costs of the guidance system. Note that the most expensive equipment is the tactile panel, which could be avoided if communication protocols of machine manufactures would be open.

The vision based incidence capture is more flexible and reliable in worst conditions (extended range \& lower curvature radius allow less repositioning of reference laser beam station), allowing saving time in comparison to conventional procedures, by increasing the distances travelled without repositioning the reference laser. With this, avoiding much influencing in the work activity is accomplished, preventing losing time, allowing a complete control of all the components of the system from a computer. An easy to handle software HMI over a tactile hardware suitable for tunnels was also implemented.

As expected, Video Target guidance systems are severely prone to problems of occlusions. With the presented system, the target's capabilities and limitations are exploited, working without needing slow and expensive relocations and recalibrations even if the machine has great deviations. A proper target installation in the setup phase of the tunneling machine causes the beam's incidence favoured and does not come out to quick from the target's range, avoiding future relocations. This minimizes the maintenance effort of a surveyor during the whole working process. Additionally, with the combination of this extended range guiding system and a proper concrete segment design, even lower curvature radius can be achieved.

\section{Acknowledgements}

This research was substantially supported by RoboCity2030-II-CM project (S2009/DPI-1559), funded by Comunidad de Madrid and co-funded by Structural Funds of the EU. Especial thanks to EUROHINCA's staff for their help in conducting field trials.

\section{References}

[1] Schneider, O., Requirements for positioning and navigation in underground constructions, In Proceedings of the International Conference on Indoor Positioning and Indoor Navigation (IPIN), Zurich, Swiss, 2010.

[2] Thomson, J., "Pipe Jacking and Microtunneling", Blackie Academic \& Professional, London, 1993.

[3] Teach. T. L, “Apparatus for method for detecting the position and orientation of a reference beam of light". Patent number: 4.786.178.Date: 11/22/1988.

[4] Stentz A, Ollis M, Scheding S., Herman H, Fromme C, Pedersen J, Hegadorn T, McCall R, Bares J, Moore R, "Position Measurement for Automated Mining Machinery", In Proceedings of the conference International Conference on Field and Service Robotics, Bruchsal Germany 1999.

[5] Hackston C, Göckel J, Messing M. "Modular concept guidance system for full coverage of pipejacking applications" VMT GmbH, In 
Proceedings of the International Conference and Exhibition on Tunnelling and Trenchless Technology, Selangor, Malaysia, 2006.

[6] Alignment Surveys Ltd. "Presentation of the TEDSS ${ }^{\circledR}$ tunnel guidance system" On-line: http://www.alignmentsurveys.com/tunnelalignment/. Accessed: 11/15/ 2013.

[7] ZED Tunnel Guidance Ltd. On-line: http://www.zed-tg.co.uk. Accessed: 11/15/ 2013

[8] VMT GmbH, "SLS-Microtunnelling LT product brochure" Bruchsal, Germany." On-line: http://vmt-gmbh.de__Accessed: 11/15/ 2013.

[9] TACS GmbH, "Summary of the ACS Guidance System", On-line: http://www.tacsgmbh.de. Accessed: 11/20/ 2013.

[10] ROBOTICS, DMP Electronics Inc. (Taipei, Taiwan) On line: http://www.roboard.com/ Accessed: 11/20/ 2013.

[11] G Bradski, Adrian Kaehler.Learning OpenCV: Computer Vision with the OpenCV Library, 2008.

[12] Metta, G.; Fitzpatrick, L. Natale, P.; Natale, L. "YARP: yet another robot platform", International Journal on Advanced Robotics Systems. 2006

[13] R. Portero, "Tunneling Boring Machine Guiadance System", Bachelor Thesis Report, Sept 2012

[14] EUROHINCA. Listado de obras ejecutadas, Online: http://www.tacsgmbh.de. Accessed: $11 / 20 / 2013$ 